\title{
The Accuracy of Point-of-Care Creatinine Testing in the Emergency Department
}

\author{
Moises Moreno, ${ }^{1}$ Adam Schwartz, ${ }^{1}$ and Ronald Dvorkin ${ }^{2}$ \\ ${ }^{1}$ Good Samaritan Hospital Medical Center, Department of Emergency Medicine, West Islip, NY 11795, USA \\ ${ }^{2}$ CityMD, Commack, NY 11725, USA
}

Correspondence should be addressed to Moises Moreno; moremois@gmail.com

Received 6 August 2015; Revised 12 November 2015; Accepted 14 November 2015

Academic Editor: Frank Hildebrand

Copyright (c) 2015 Moises Moreno et al. This is an open access article distributed under the Creative Commons Attribution License, which permits unrestricted use, distribution, and reproduction in any medium, provided the original work is properly cited.

\begin{abstract}
Objective. To determine the accuracy of Point-Of-Care testing (PoCT) creatinine values when compared to standard central laboratory testing (IDMS) and to demonstrate if and how a discrepancy could lead to improper risk stratification for contrast induced nephropathy (CIN). Methods. We conducted a descriptive retrospective chart review of patients seen in the Emergency Department of a single suburban, community, and academic medical center. We included patients who presented to the department between March 2013 and September 2014 who had blood samples analyzed by both PoCT and IDMS. Results. Mean IDMS creatinine values were $0.23 \mathrm{mg} / \mathrm{dL}$ higher when compared with i-Stat values. $95 \%$ of the time, the IDMS creatinine value was variable and ranged from $-0.45 \mathrm{mg} / \mathrm{dL}$ to $+0.91 \mathrm{mg} / \mathrm{dL}$ when compared to the i-Stat creatinine. When using i-Stat creatinine values to calculate GFR, 47 out of 156 patients had risk category variations compared to using the IDMS value. This affected $30.1 \%$ of the total eligible sample population (22.9\% to $37.3 \%$ with $95 \%$ CI). Conclusion. We found a significant discrepancy between PoCT and IDMS creatinine values and found that this discrepancy could lead to improper risk stratification for CIN.
\end{abstract}

\section{Introduction/Background}

Contrast-induced nephropathy (CIN) is a known complication of procedures in which intravascular iodinated contrast media are administrated $[1,2]$. Patients with preexisting renal impairment have an increased risk of developing CIN. Previous studies have demonstrated that a glomerular filtration rate (GFR) $<60$ is a risk factor for CIN [3]. Creatinine value is an essential component when determining GFR. It is important to rapidly identify patients who have elevated creatinine to risk-stratify patients before administrating iodinated contrast media.

Currently, the gold standard to accurately measure creatinine in the emergency department $(\mathrm{ED})$ is by isotope dilution mass spectrometry (IDMS) in a central laboratory. Ideally, this test can take at least thirty minutes for the laboratory to analyze. However, in reality, it can take even longer to obtain the value in a busy ED. During critical situations, obtaining the creatinine rapidly would allow the physician to determine the best diagnostic testing or therapy for a patient to prevent CIN. As an example, we feel that if we could use point-of-care testing (PoCT) rather than sending the samples to the central laboratory, this could expedite decision making in traumas and critical patients. PoCT with a blood analyzer such as the $\mathrm{i}$-Stat system is being used to provide real-time results in the ED within minutes $[2,4]$.

If PoCT is as accurate as the IDMS from the central laboratory, using PoCT in these situations could allow the ED physician to quickly send a patient to CT scan for the appropriate study without delay.

During the past 18 months, our department obtained over 724 PoCT chemistry studies. Subjectively, many providers recall instances where the resulting i-Stat creatinine values were significantly different compared to the values from the central laboratory testing.

It is important to determine the accuracy of PoCT testing in real time since inaccurate PoCT testing will impact patient care. According to data published on their website, the PoCT system, the Chem8+, designed by Abbott, Point of Care, Inc., was verified independently and approved for use by the FDA in 2006. They verified their machine's accuracy using linear regression analysis which demonstrated correlations 
all greater than 0.985 . They also verified the precision of their system by testing multiple cartridges for 39 patient samples and showed the average creatinine difference across all reference ranges to be less than $0.04 \mathrm{mg} / \mathrm{dL}$. According to their study, they noted specifically that acetaminophen, acetylcysteine, ascorbate, bromide, creatine, glycolic acid, and hydroxyurea could affect their Chem8+ values. This study was done in a controlled environment and we sought to determine if the results are still accurate in real-time use in an emergency department. If a physician uses an erroneous lab value for medical decision making, it could place a patient at higher risk for developing CIN or delay the physician from ordering an appropriate study.

\section{Hypothesis}

The creatinine measurement obtained via i-Stat PoCT does not equal the values obtained via IDMS standard laboratory testing.

\section{Methods and Study Design}

This study is a descriptive retrospective chart review of patients seen in the Emergency Department of a single suburban, community, academic medical center with an annual census of approximately 98,000 patients. The study includes patients who presented to the department from March 2013 (the implementation of our new EMR) to September 2014 who had blood samples analyzed by both i-Stat testing and the central laboratory IDMS. We investigated the measurement agreement between the PoCT i-Stat creatinine values and those from the central laboratory IDMS testing and how their discrepancy affected changes in the GFR risk categories.

In our department, respiratory therapists, who are certified by the central laboratory, perform PoCT. The PoCT used in this study is the Chem8+, which measures serum sodium, potassium, chloride, bicarbonate, anion gap, ionized calcium, glucose, urea nitrogen, hemoglobin, hematocrit, and creatinine. Since the creatinine level can change dynamically, we only included patients who had an i-Stat serum test performed within ten minutes of retrieving blood sent to the standard laboratory. Exclusion criteria included patients with missing or incomplete charts, patients not originally evaluated in the ED, and patients who had blood chemistries drawn outside of ten minutes of the i-Stat sample.

The data was abstracted from Abbott's precision web point-of-care management system. All of the patients seen in the ED who had a PoCT i-Stat performed from March 2013 to September 2014 were enrolled. Their records were manually reviewed through our electronic medical record system (Epic System Hyperspace 2014 IU 1). The Epic and Abbott Precision Web system made it possible to include all eligible patients and analyze all data points including age, sex, weight, collection times, and sample type (arterial or venous). Once the patient population was obtained, the creatinine results via PoCT were compared to the creatinine results via standard laboratory testing. In addition, for data analysis purposes, we calculated the GFR for patients using the creatinine obtained from both samples using the Cockcroft-Gault formula [5],
TABLE 1: Characteristics of $156 \mathrm{ED}$ visits where patients received creatinine testing from i-Stat and laboratory methods within 10 minutes of each other during visits over 18-month period between March 2013 and September 2014.

\begin{tabular}{lc}
\hline Demographics & $\begin{array}{c}\text { Study group } \\
n=156\end{array}$ \\
\hline Average age, years (SD) & $62.3(21.0)$ \\
Average weight, kg (SD) & $80.4(23.3)$ \\
Gender \%F (CI) & $46.2 \%(38.33$ to 53.97$)$ \\
\hline
\end{tabular}

SD: Standard deviation; CI: confidence interval.

TABLE 2: Sample discrepancy between the two methods of measurement.

\begin{tabular}{lcc}
\hline PoCT Cr > IDMS Cr & PoCT Cr < IDMS Cr & PoCT Cr $=$ IDMS Cr \\
\hline 34 & 114 & 8
\end{tabular}

PoCT: point-of-care testing; Cr: creatinine; IDMS: isotope dilution mass spectrometry.

which is the formula our institution currently uses to accept patients for CT studies with IV contrast. Patients with a GFR $<60$ are not recommended to receive IV contrast studies. Private health information (PHI) was initially obtained but not stored in the study. The charts were only searched for collection times, sample type (arterial or venous), demographics, and creatinine value.

All statistical analysis was performed by use of a commercially available statistical program (Excel Microsoft 2008 and Excel Analyze-it 2007 Method Validation Edition).

\section{Results}

A total of 724 i-Stat creatinine tests were performed between March 2013 and September 2014 in the ED. The demographics are listed in Table 1 . We excluded 568 samples from the study because of exclusion criteria. Of the 724 samples obtained over our study time frame, 156 patients were eligible for the study as shown in Figure 1.

All samples were obtained within 10 minutes of each other ( median $=5$ minutes; IQR $=2$ to 8 minutes). Calculations for all of our samples concluded that, on average, IDMS samples had a creatinine value $0.23 \mathrm{mg} / \mathrm{dL}$ higher than PoCT samples. Only eight eligible patients had the same creatinine value for both samples. In 114 cases, the IDMS measurement was higher, and in 34 cases it was lower than the i-Stat PoCT value (as shown in Table 2).

In this study, we are interested in the accuracy of PoCT creatinine testing compared to IDMS creatinine. We used a Bland-Altman plot $[6,7]$ to analyze the agreement between the two values. The Bland-Altman method plot demonstrates the agreement between two methods of measurement by plotting the hypothetical true value (average of the two methods) against the difference of the two measurements. Figure 2 demonstrates the agreement between PoCT i-Stat creatinine values and the IDMS values in our study.

The plot shows a trend of positive values for IDMS creatinine and i-Stat creatinine. This implies that IDMS 


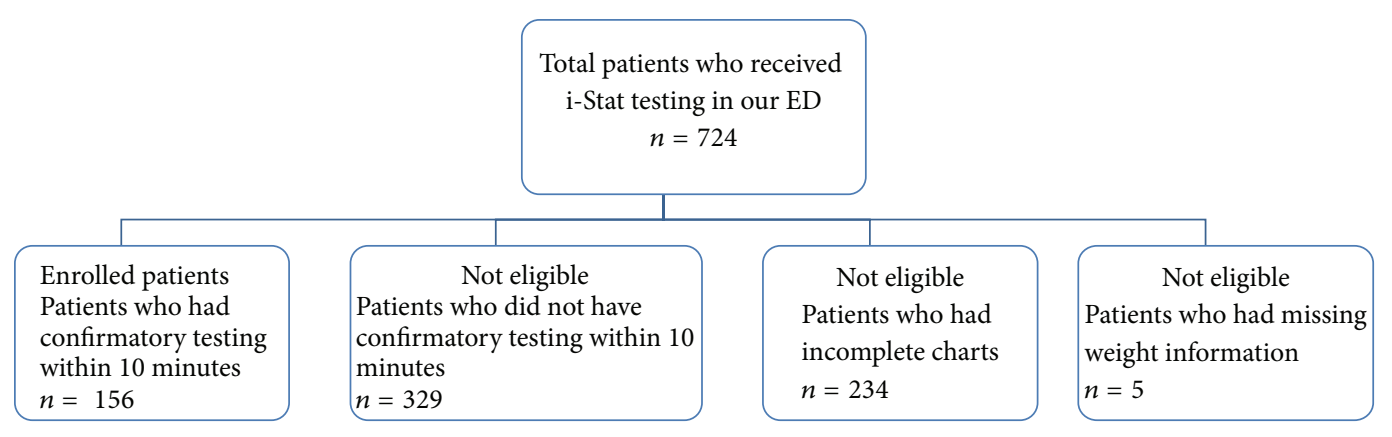

FIgURE 1: Flow diagram of the selection process for final study population.

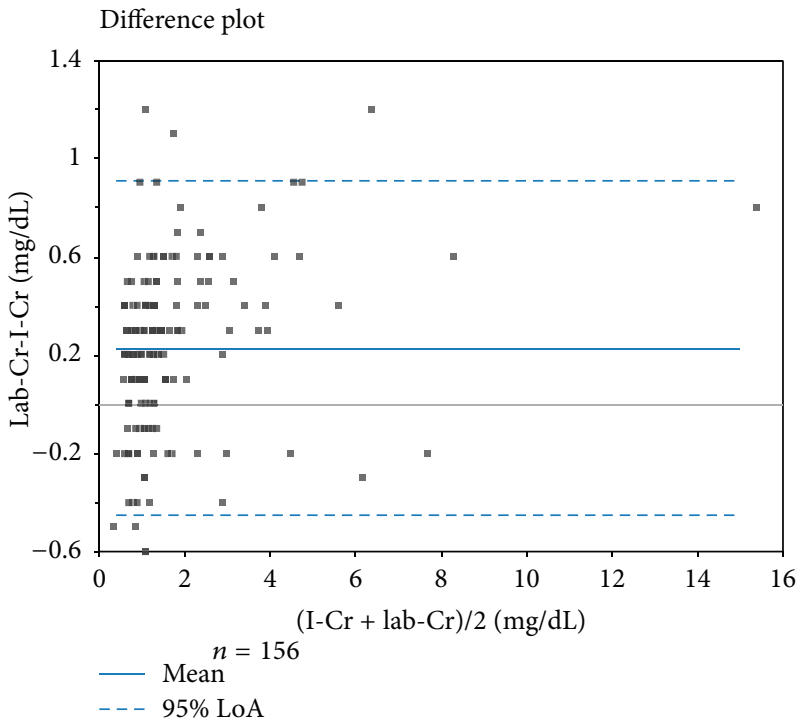

FIGURE 2: Data plotted using Band-Altman method to show the difference between the two methods against the average measurement. Cr: creatinine.

creatinine tends to have higher values. Also we can see how most outliers stand on the extremes of the difference.

The central laboratory creatinine values had a measurement interval of $0.4 \mathrm{mg} / \mathrm{dL}$ to $15 \mathrm{mg} / \mathrm{dL}$, while those of the i-Stat testing had a measurement interval of $0.1 \mathrm{mg} / \mathrm{dL}$ to $15.8 \mathrm{mg} / \mathrm{dL}$. The laboratory creatinine average was $1.84 \mathrm{mg} / \mathrm{dL}$ while the i-Stat creatinine average was $1.61 \mathrm{mg} / \mathrm{dL}$. The BlandAltman test found that the mean value of the difference between the two measurements is $0.23 \mathrm{mg} / \mathrm{dL}$ and since it is different than a perfect agreement of 0 , it is considered a fixed bias between two methods. The limits of agreement were calculated as the bias \pm 1.96 standard deviations of 0.35 , which calculates the difference value between the creatinine obtained by both measurements to range from $-0.45 \mathrm{mg} / \mathrm{dL}$ to $0.91 \mathrm{mg} / \mathrm{dL}$. This range accounts for fixed bias as well as error measurement. Accuracy was defined as the 95\% confidence interval of the limits of agreement, which corresponds with statistical normal distribution avoiding measurement error.

The patients' GFRs were calculated according to the Cockcroft-Gault equation. These values were then categorized according to the levels of risk following the Guidelines for Contrast Administration from the American College of Radiology [8-13]. The four categories consist of high, intermediate, low, and negligible risk. The high risk category includes GFR values less than $30 \mathrm{~mL} / \mathrm{min} / 1.73 \mathrm{~m}^{2}$; the intermediate risk category includes GFR values between 30 and $44 \mathrm{~mL} / \mathrm{min} / 1.73 \mathrm{~m}^{2}$; the low risk category includes GFR values between 45 and $60 \mathrm{~mL} / \mathrm{min} / 1.73 \mathrm{~m}^{2}$; the negligible risk category includes GFR values higher than $60 \mathrm{~mL} / \mathrm{min} / 1.73 \mathrm{~m}^{2}$. We then calculated the number of patients whose level of risk was not accurately predetermined when their GFR was calculated using the PoCT creatinine value versus their IDMS value. Even though most of the patients' creatinine changed, the level of risk remained in the same category for 109 patients, 9 patients' risk category decreased, and 38 patients' risk category increased when using their i-Stat creatinine value versus their IDMS creatinine value. Thirty-eight out of those 47 patients $(80.9 \%, 99 \%$ CI 66.1 to $95.6 \%)$ were actually at a higher risk category for CIN. This data is displayed in Tables 3(a) and 3(b).

\section{Discussion}

Physicians in our ED have the ability to obtain PoCT creatinine values. These results are often available much sooner than standard IDMS testing, which must be sent to the hospital's central laboratory. Creatinine is a well-established marker for renal function and it is widely used to estimate the GFR [14]. GFR is used to risk-stratify patients for their ability to tolerate IV contrast for CT scans. Often, IV contrast CT scans are the ideal study to rule out significant pathology in critically ill patients. Therefore, obtaining an accurate value of a patient's creatinine is imperative to the physician's diagnosis. There were many anecdotal reports from the ED physicians in our ED noting discrepancies between creatinine values obtained via PoCT testing and IDMS testing. Our study analyzed if in fact there are significant differences in values and if the values altered the patients' risk stratification.

Our data analysis revealed the following.

(1) Based upon our results, 95\% of the time, the IDMS creatinine value was variable and could be either $0.45 \mathrm{mg} / \mathrm{dL}$ below or $0.91 \mathrm{mg} / \mathrm{dL}$ above the i-Stat creatinine. In our emergency department, on average, IDMS creatinine is $0.23 \mathrm{mg} / \mathrm{dL}$ higher than the one from the i-Stat testing. 
TABLE 3: (a) The changes in risk categories when comparing GFR obtained initially from PoCT to the IMDS central lab. (b) This table is a summary of Table 3(a). The number of patients who had a change in GFR risk stratification when using PoCT i-Stat versus IDMS central lab. Of the 47 significant discrepancies, $80.6 \%$ would have underclassified the risk of CIN (80.9\%, 99\% CI 66.1 to $95.6 \%)$.

(a)

\begin{tabular}{lcccc}
\hline \multirow{2}{*}{ PoCT i-Stat } & & \multicolumn{2}{c}{ IDMS central lab (control) } & \\
& Resulted in high & Resulted in intermediate & Resulted in low & Resulted in negligible \\
\hline Initially high & 30 & $\mathbf{0}$ & $\mathbf{0}$ & $\mathbf{0}$ \\
Initially intermediate & $\mathbf{4}$ & 8 & $\mathbf{1}$ & $\mathbf{1}$ \\
Initially low & $\mathbf{2}$ & $\mathbf{1 1}$ & 3 & $\mathbf{7}$ \\
Initially negligible & $\mathbf{0}$ & $\mathbf{3}$ & $\mathbf{1 8}$ & 68 \\
\hline
\end{tabular}

PoCT: point-of-care testing; GFR: glomerular filtration rate; IDMS: isotope dilution mass spectrometry.

(b)

PoCT i-Stat GFR values compared to actual IDMS central lab GFR values

\begin{tabular}{lcc} 
Risk decreased & Risk increased & Risk remained the same \\
\hline $\mathbf{9}$ & $\mathbf{3 8}$ & $\mathbf{1 0 9}$
\end{tabular}

PoCT: point-of-care testing; GFR: glomerular filtration rate; IDMS: isotope dilution mass spectrometry; CIN: contrast-induced nephropathy.

Calculations reveal an average of $22 \%$ difference between IDMS and the i-Stat creatinine values, which is significantly higher than the desirable analytical goal of $8.87 \%$ total error for creatinine serum as indicated by Westgard [14]. This variability, fixed bias, and percentage of difference limit the ability of the physicians in our ED to make a reliable, well-informed decision on whether or not to order a CT scan with contrast.

(2) When using i-Stat creatinine values to calculate GFR, 47 out of 156 patients had risk category variations. Those are equal to $30.1 \%$ of total eligible sample population $(22.9 \%$ to $37.3 \%$ with $95 \%$ CI). 38 out of those 47 patients $(80.9 \%, 99 \%$ CI 66.1 to $95.6 \%$ ) were actually at a higher risk category for CIN.

For the patients eligible for inclusion in our study, almost one-fourth of them would have increased risk for IV contrastinduced nephropathy if their GFRs were calculated using creatinine values from IDMS testing versus those from PoCT testing. This is a significant cause for concern and places a significant percentage of patients at risk.

In addition, if GFRs were calculated using PoCT creatinine values, rather than IDMS creatinine values, 9 out of 156 patients $(6 \%)$ may have been eligible for IV contrast based on GFR risk stratification and not received these studies due to underestimation of their GFR. This could lead to a potential delay in obtaining the ideal radiologic study and potentially lead to repeat imaging with IV contrast, which exposes the patient to additional radiation. Therefore, basing GFR calculations on i-Stat creatinine in our ED could mean wrongly risk-stratifying the patients.

We know that neither of the previously mentioned scenarios is acceptable in our ED. Qualitatively our study demonstrates that our ED physicians should not use only PoCT creatinine values to risk-stratify patients for CT imaging studies if creatinine values are within the variation range.

Every six months, i-Stat receives validations by two regulatory agencies, JACHO and NYS. The FDA has reviewed iStat Chem8+ and CLEA has waived it [15]. However, the tests were only run on potassium. In addition, Abbott participates in an external proficiency testing three times a year, even though they only need an $80 \%$ match to pass. The i-Stat manual describes a thorough process to generate creatinine values [16]. We know that i-Stat PoCT is a highly convenient device and we know that there is a high correlation of 0.98 between the two measurements. However, in our ED, the variation is higher than desirable and therefore caution should be used for decision making when relying on the PoCT.

Overall, this study is concerning for the accuracy of PoCT creatinine testing. Our results seem to demonstrate a difference from the published data for accuracy from Abbott. It is unclear what is causing the difference in creatinine values. Potential causes include therapist technique, type of syringes used, venous versus arterial samples, inaccurate calibration by the hospital laboratory, and test inaccuracy. Future followup studies are needed to investigate the source of these inaccuracies and offer possible suggestions on improving such a convenient and needed device as the i-Stat in EDs. Our study also did not look at the accuracy of other values obtained on the i-Stat such as hemoglobin, hematocrit, and potassium or the repeatability of the measurements for the same patients. We hope that a future study will look into the accuracy of those values as well.

\section{Summary}

In conclusion, we analyzed the accuracy of PoCT creatinine testing compared to IDMS creatinine. We found significant discrepancy between the two methods of measurement in our controlled sample population. The clinical significance of these discrepancies should be taken into consideration when point-of-care testing is performed. There may be a potential to misclassify patient risk when utilizing PoCT.

\section{Limitations}

This study has multiple limitations. It is limited by being based at a single center, which is a suburban, community 
based, and academic emergency department. The results from this study are not necessarily representative of other centers. The study was limited to a single PoCT device used in our ED. The results are also limited by the techniques of the respiratory therapists who performed the studies. We also only had 156 of 724 patients who met all inclusion criteria. There are limitations in calculating the GFR because of patient weight. We assume all weights to be accurate. However, many weights in our department are estimations. It is unclear if this would have any significant difference on the true change in GFR. Our study is also limited by the fact that most patients in our department, who have iStats obtained, are critically ill patients and the results may not be representative of the overall demographics of our department. This study was also limited by the fact that we did not look at outcomes as correlated with the GFR and the study was underpowered to look at correlations of discrepancies with patient age. Another assumption made in this study is that we hold the laboratory as the standard measure and consider it to reveal the true value of creatinine. However, it was possible that the laboratory's calibration methods lacked the required degree of accuracy. This study did not attempt to quantify the actual risk to patients as there is no universal quantifiable risk identified. The study was not designed to find trends on admissions based on GFR.

\section{Conflict of Interests}

The authors declare that there is no conflict of interests regarding the publication of this paper.

\section{References}

[1] J. S. You, Y. E. Chung, J. W. Park et al., "The usefulness of rapid point-of-care creatinine testing for the prevention of contrast-induced nephropathy in the emergency department," Emergency Medicine Journal, vol. 30, no. 7, pp. 555-558, 2013.

[2] F. Martínez Lomakin and C. Tobar, "Accuracy of point-ofcare serum creatinine devices for detecting patients at risk of contrast-induced nephropathy: a critical overview," Critical Reviews in Clinical Laboratory Sciences, vol. 51, no. 6, pp. 332343, 2014.

[3] S. J. Traub, J. A. Kellum, A. Tang, L. Cataldo, A. Kancharla, and N. I. Shapiro, "Risk factors for radiocontrast nephropathy after emergency department contrast-enhanced computerized tomography," Academic Emergency Medicine, vol. 20, no. 1, pp. 40-45, 2013.

[4] C. Papadea, J. Foster, S. Grant et al., "Evaluation of the i-STAT portable clinical analyzer for point-of-care blood testing in the intensive care units of a university children's hospital," Annals of Clinical \& Laboratory Science, vol. 32, no. 3, pp. 231-243, 2002.

[5] D. W. Cockcroft and M. H. Gault, "Prediction of creatinine clearance from serum creatinine," Nephron, vol. 16, no. 1, pp. 3141, 1976.

[6] J. M. Bland and D. G. Altman, "Statistical methods for assessing agreement between two methods of clinical measurement," The Lancet, vol. 1, no. 8476, pp. 307-310, 1986.

[7] J. M. Bland and D. G. Altman, "Measuring agreement in method comparison studies," Statistical Methods in Medical Research, vol. 8, no. 2, pp. 135-160, 1999.
[8] ACR Committee on Drugs and Contrast Media, "Contrastinduced nephrotoxicity," in ACR Manual on Contrast Media, Version 9, chapter 8, ACR Committee on Drugs and Contrast Media, 2013.

[9] S. Hiremath, A. Akbari, W. Shabana, D. A. Fergusson, and G. A. Knoll, "Prevention of contrast-induced acute kidney injury: is simple oral hydration similar to intravenous? A systematic review of the evidence," PLoS ONE, vol. 8, no. 3, Article ID e60009, 2013.

[10] S. M. Kim, R.-H. Cha, J. P. Lee et al., "Incidence and outcomes of contrast-induced nephropathy after computed tomography in patients with CKD: a quality improvement report," American Journal of Kidney Diseases, vol. 55, no. 6, pp. 1018-1025, 2010.

[11] S. K. Morcos, H. S. Thomsen, and J. A. W. Webb, "Contrastmedia-induced nephrotoxicity: a consensus report," European Radiology, vol. 9, no. 8, pp. 1602-1613, 1999.

[12] A. J. Taylor, D. Hotchkiss, R. W. Morse, and J. McCabe, "PREPARED: preparation for angiography in renal dysfunction: a randomized trial of inpatient vs outpatient hydration protocols for cardiac catheterization in mild-to-moderate renal dysfunction," Chest, vol. 114, no. 6, pp. 1570-1574, 1998.

[13] S. D. Weisbord and P. M. Palevsky, "Prevention of contrastinduced nephropathy with volume expansion," Clinical Journal of the American Society of Nephrology, vol. 3, no. 1, pp. 273-280, 2008.

[14] J. Westgard, "Desirable specifications for total error, imprecision, and bias, derived from intra- and inter- individual biological variation," March 2015, http://westgard.com/biodatabase1 .htm.

[15] US Food and Drug Administration, "Clinical Laboratory Improvement Amendments," March 2015, http://www.accessdata.fda.gov/scripts/cdrh/cfdocs/cfCLIA/Detail.cfm?ID=28125.

[16] Abbot Point of Care, Procedure Manual for the i-Stat System, November 2014, http://viewppt.com/docs/www__med__navy_mil-sites-nmotc-nemti-Documents-Tech\%20Online-LABORATORY\%20EQUIPMENT-Istat\%20Procedure_Manual_ jan\%20\%2016\%202012-\%20714446-00H_-pdf.html. 


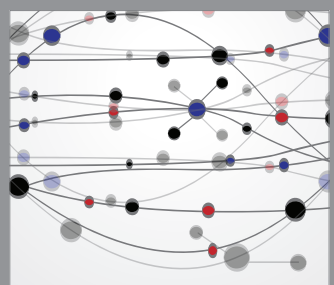

The Scientific World Journal
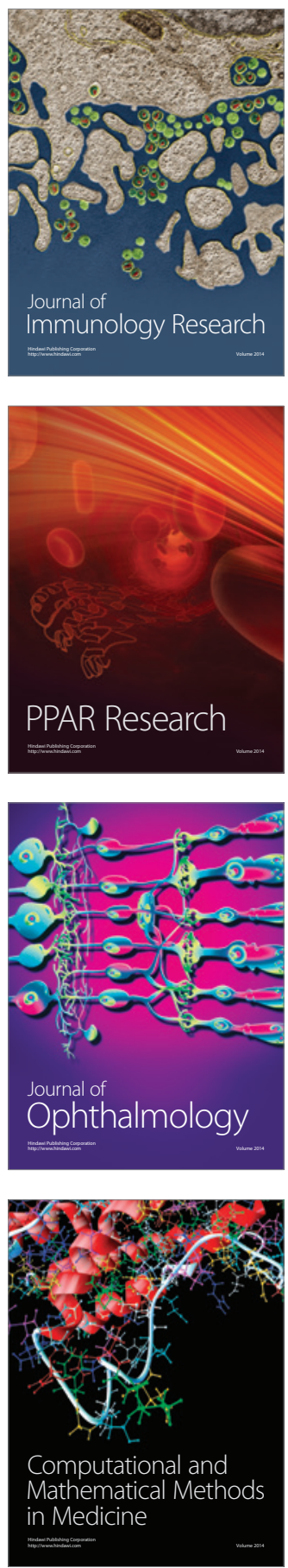

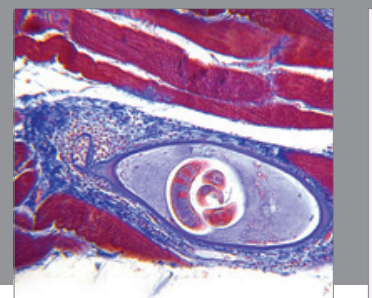

Gastroenterology

Research and Practice
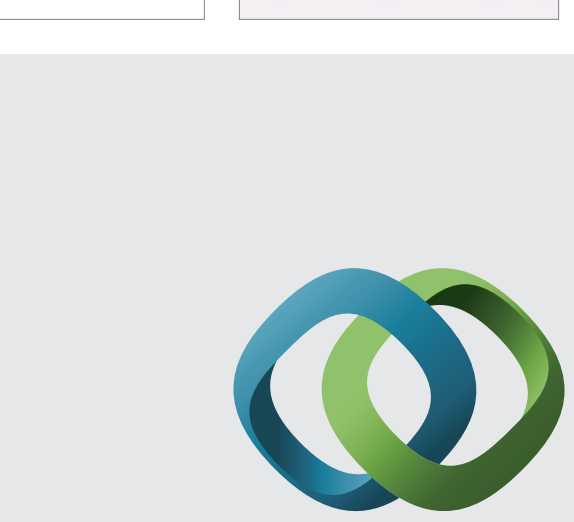

\section{Hindawi}

Submit your manuscripts at

http://www.hindawi.com
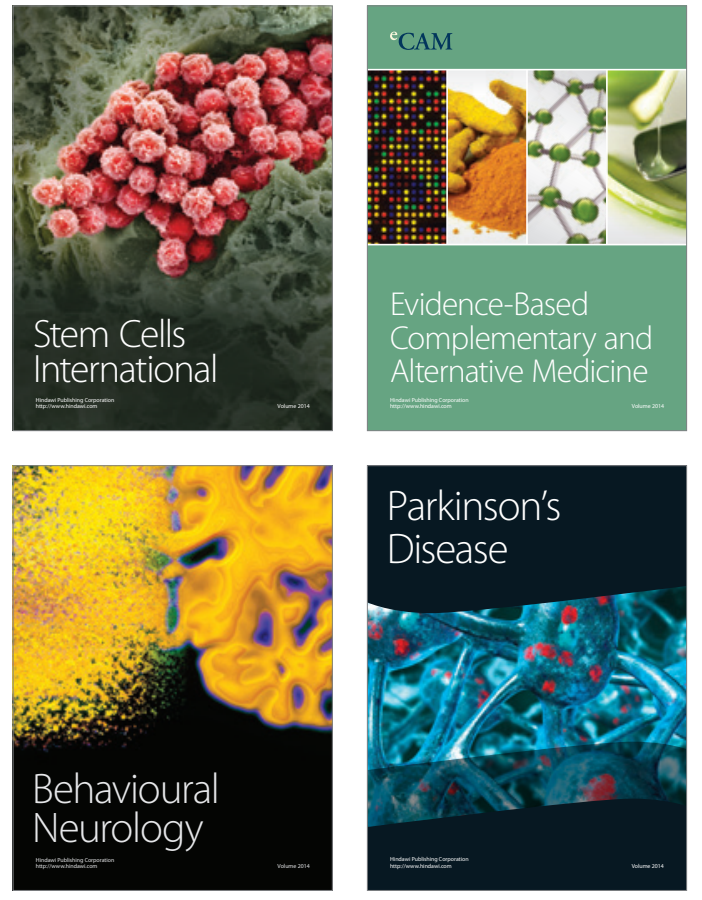
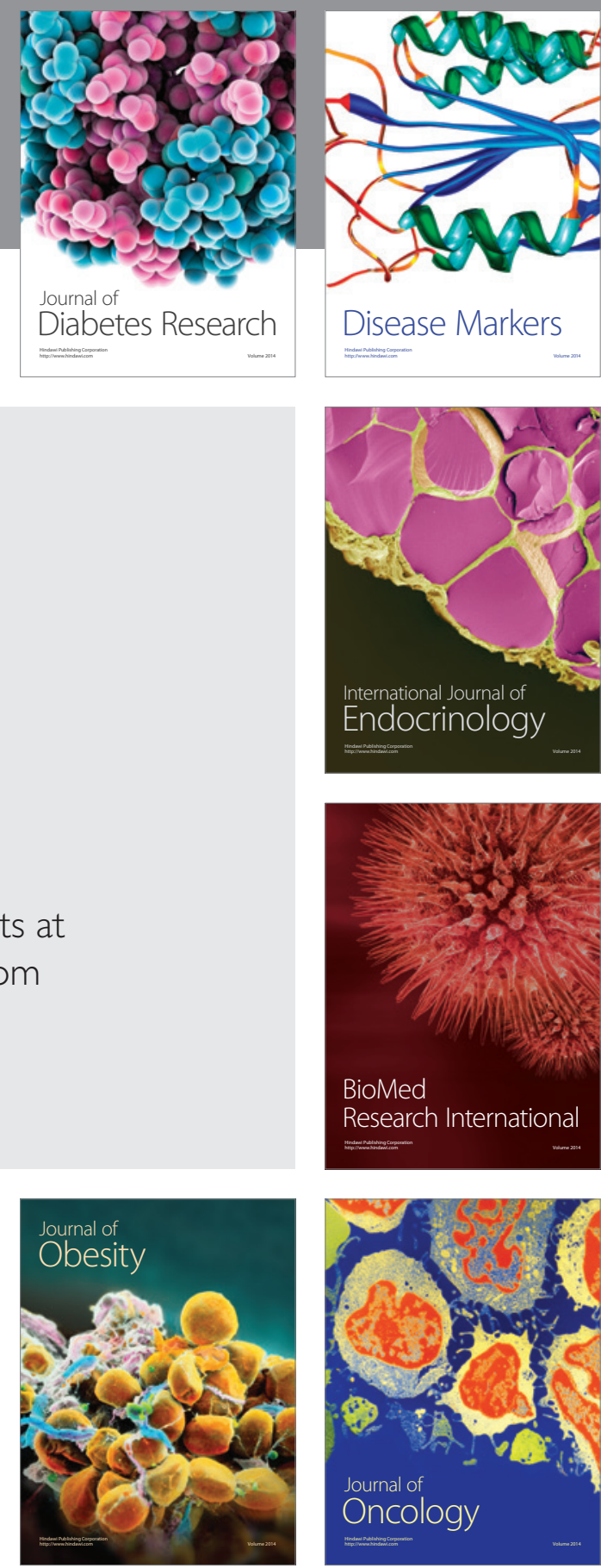

Disease Markers
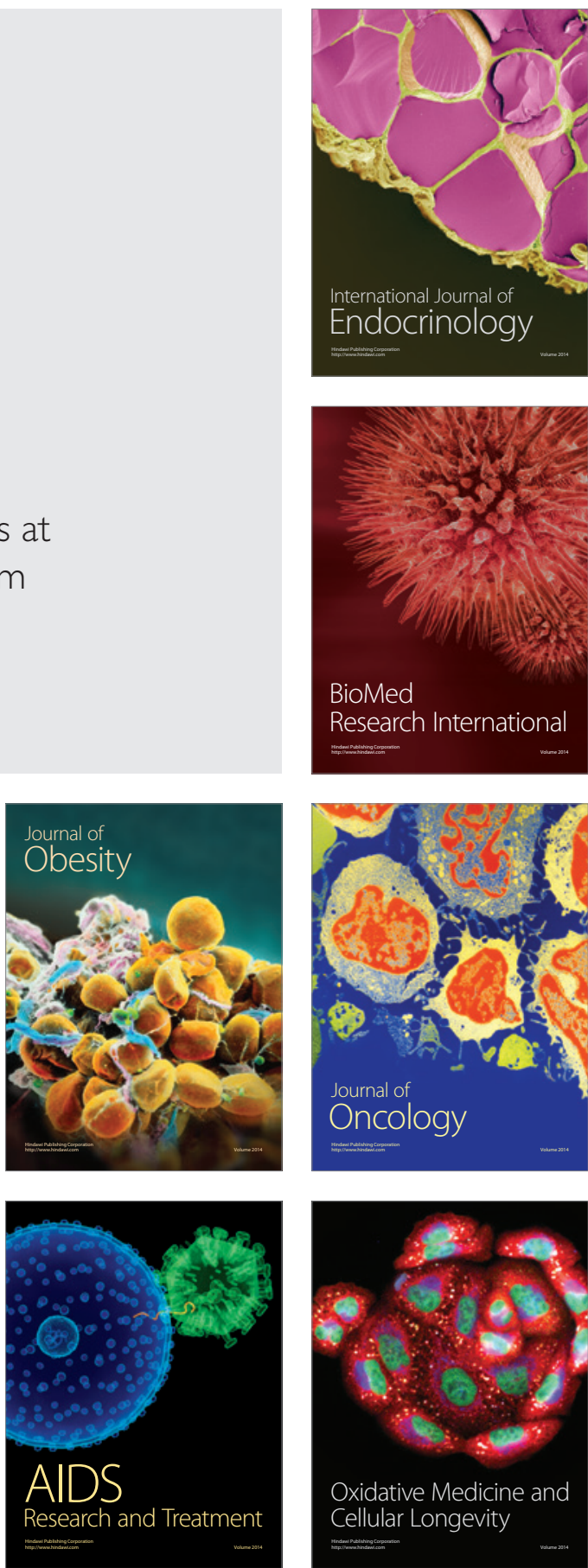